\section{Clasificación según nivel de morbilidad e intensidad del uso de recursos de una población con patologías crónicas en Atención Primaria de Salud utilizando "Adjusted Clinical Groups" (ACG)}

\author{
EMILIO SANTELICES C. ${ }^{1, \mathrm{a}}$, FERNANDO MUÑOZ P. ${ }^{2, \mathrm{~b}}$, \\ PATRICIO MUÑIZ ${ }^{3}$, JOSÉ ROJAS ${ }^{1, \mathrm{c}}$
}

\section{Adjusted Clinical Groups Method (ACG) to allocate resources according to the disease burden of each health center}

Background: Health care must be provided with strong primary health care models, emphasizing prevention and a continued, integrated and interdisciplinary care. Tools should be used to allow a better planning and more efficient use of resources. Aim: To assess risk adjustment methodologies, such as the Adjusted Clinical Groups (ACG) developed by The Johns Hopkins University, to allow the identification of chronic condition patterns and allocate resources accordingly. Material and Methods: We report the results obtained applying the ACG methodology in primary care systems of 22 counties for three chronic diseases, namely Diabetes Mellitus, Hypertension and Heart Failure. Results: The outcomes show a great variability in the prevalence of these conditions in the different health centers. There is also a great diversity in the use of resources for a given condition in the different health care centers. Conclusions: This methodology should contribute to a better distribution of health care resources, which should be based on the disease burden of each health care center.

(Rev Med Chile 2016; 144: 291-297)

Key words: Delivery of Health Care, Integrated; Primary Health Care; Resource Allocation; Risk Adjustment.

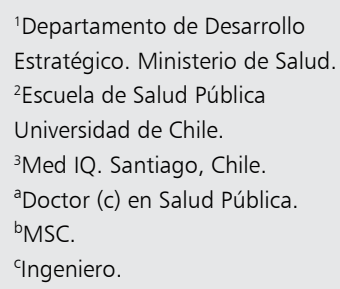

Recibido el 5 de agosto de 2015, aceptado el 10 de diciembre de 2015

Correspondencia a:

Emilio Santelices Cuevas

Lo Fontecilla 441, Las Condes.

esanteli@clc.cl
4 1 uso con racionalidad de los diferentes mecanismos de pago existentes es una de las herramientas más poderosas que debe manejar hoy un gerente o directivo de salud. Según sea el mecanismo utilizado, se afectará el comportamiento de financiadores y prestadores ${ }^{1}$.

El interés del estudio de los efectos de estos mecanismos radica en la importancia de identificar, a través de la evidencia empírica, la forma en que pueden afectar los comportamientos de los financiadores o proveedores e impactar a los sistemas de salud, en relación a la eficiencia en el uso de los recursos y en la calidad en la provisión de los servicios ${ }^{2}$.

En Chile, la asignación de recursos para la Atención Primaria de Salud (APS) ha evolucionado desde un mecanismo de pago por acto ( fee for service) a un mecanismo per cápita ajustado por condiciones de ruralidad y pobreza. El mecanismo inicial, denominado FAPEM (Facturación por Atención Prestada en Establecimientos Municipales) incentivaba la actividad en aquellas 
prestaciones que dejaban excedente y que no resultaban necesarias, razón por la que a poco andar, se estableció un techo máximo. Este sistema recibió críticas, pues ni siquiera suministraba incentivos a los resultados ${ }^{3}$.

Más tarde, se ideó un mecanismo de pago per cápita que coexiste con el financiamiento prospectivo de programas prioritarios definidos por el Ministerio de Salud en su nivel central. Se esperaba que este mecanismo incentivara las actividades de prevención en la gestión de la APS municipal.

El sistema de pago per cápita vigente está sujeto a una serie de indexadores relacionados con el nivel socioeconómico de la comuna, ruralidad, dificultad de acceso y otras variables que dan cuenta de la dificultad de la gestión en algunas comunas respecto al promedio.

Cabe destacar que si bien los criterios de indexación del per cápita basal son claros, para su definición se utilizan promedios históricos de demanda esperada, los que de acuerdo a algunos autores no resultan precisos ${ }^{4}$.

El mecanismo de asignación utilizado en la actualidad ha permitido resolver las dificultades observadas con los pagos históricos, sin embargo, no permite ajustar la distribución de los recursos considerando el perfil epidemiológico de la población, el que se continuará modificando en los próximos años.

La ausencia de mecanismos que den cuenta de la distribución de los recursos en función de la carga de enfermedad de la población, contribuye estructuralmente a perpetuar la inequidad. Las dos últimas encuestas nacionales de salud realizadas en nuestro país indican que los pacientes con enfermedades crónicas se distribuyen con mayor prevalencia en aquellos grupos con menor nivel educacional ${ }^{5}$.

Estos antecedentes han motivado estudios orientados, en primer término, a validar el uso de Grupos Clínicos Ajustados (ACG) para la población chilena y a identificar los patrones de carga de morbilidad y consumo de recursos en la población. Santelices et al. ${ }^{6}$ utilizan la información de los registros electrónicos para hacer una caracterización de la carga de morbilidad en la población asignada a centros de atención de salud primaria, encontrando evidencia significativa de la relación entre nivel de morbilidad y consumo de recursos.

Adicionalmente, utilizando el sistema Grupos Clínicos Ajustados o "Adjusted Clinical Groups"
(ACG) de la Universidad de Johns Hopkins ${ }^{7}$ se presenta un método de distribución de recursos considerando las diferencias en carga de morbilidad. Desde esta perspectiva, nos ha parecido de interés compartir la experiencia de financiamiento de la APS chilena y los resultados de los estudios que hemos realizado en materia de ajuste de riesgo para el pago capitado ${ }^{8}$.

El objetivo de este trabajo es estudiar la distribución de la población con enfermedades crónicas y su composición según la complejidad requerida para abordarlas, en un grupo de comunas seleccionadas de Chile.

\section{Metodología}

\section{Aplicación de la herramienta ACG}

El sistema Grupos Clínicos Ajustados es una herramienta de clasificación de pacientes según carga de morbilidad. Para la clasificación se consideran la edad y sexo de los pacientes en conjunto con todos los diagnósticos registrados en un período de tiempo determinado, que comúnmente es de un año. En función a estas variables, cada paciente es asignado a una de 300 categorías mutuamente excluyentes, éstas se caracterizan por poseer un peso relativo de acuerdo al consumo de recursos que los pacientes asignados a ella hicieron en relación al consumo promedio total. Si una categoría ACG posee un peso relativo de 1, implica que el consumo de recursos de los pacientes incluidos en ella es equivalente al consumo promedio total de todos los pacientes. La asignación de pesos relativos permite agrupar pacientes según la intensidad de uso de recursos que utilizan, la herramienta ACG denomina a estos grupos como "Resource Utilization Bands" (RUB). Para el caso particular de este estudio define tres de ellas, ordenadas de menor a mayor intensidad de uso de recursos.

\section{Selección de la muestra}

La muestra comprende los meses de enero a diciembre de 2012 y contiene un universo de 5.961.422 de pacientes atendidos en 270 centros de salud de atención primaria en 111 comunas que contaban con registro clínico electrónico. El identificador de los pacientes fue encriptado previamente para evitar su identificación, pero manteniendo la capacidad de identificar múltiples visitas de un mismo paciente. En la Tabla 1 se presenta el reporte con los diagnósticos procesados 
Tabla 1. Reporte de cantidad de diagnósticos procesados

\begin{tabular}{|ll|}
\hline Reporte & Valor \\
\hline Número de diagnósticos por pacientes inscritos & 2,07 \\
\hline Número de diagnósticos por pacientes atendidos & 4,49 \\
\hline Número de diagnósticos no reconocidos & 5 \\
\hline
\end{tabular}

por pacientes, la referencia para este indicador es de 6 diagnósticos por persona atendida ${ }^{7}$.

Posteriormente, se realizó un ajuste de representatividad de la muestra que consistió en seleccionar las comunas que tuvieran la mayor cantidad de centros de atención primaria (Centro de Salud Familiar o CESFAM, Consultorio General Rurales o CGR y Consultorio General Urbanos o CGU) cuya información es procesada en el sistema ACG versus las registradas en el Departamento de Es- tadísticas e Información del Ministerio de Salud (DEIS), quedando una cantidad de 1.876 .532 pacientes, en 64 centros de atención primaria, distribuidos en 22 comunas (Tabla 2).

Para este estudio sólo se consideraron las patologías: diabetes mellitus (diabetes), hipertensión arterial (HTA) e insuficiencia cardiaca (Ins. Cardiaca). Los agrupadores ACG permiten determinar si un paciente posee alguna de estas patologías sobre la base de los diagnósticos y los fármacos que se le prescriban.

\section{Resultados}

Para cada patología se presentan tres tipos de distribución de casos; en primer lugar se presenta la prevalencia en el total de la muestra por cada mil habitantes, en segundo lugar se muestra una comparación de la morbilidad relativa (los pesos relativos generales) para cada patología y, final-

Tabla 2. Número de CESFAM por comunas, en registro DEIS y número de comunas registradas en estudio con el total de población de cada una de ellas

\begin{tabular}{|c|c|c|c|c|}
\hline Glosa ACG & Glosa DEIS & n ACG & n DEIS & n pacientes \\
\hline Caldera & Caldera & 1 & 1 & 18.256 \\
\hline Catemu & Catemu & 1 & 1 & 12.434 \\
\hline Cerro Navia & Cerro Navia & 4 & 4 & 155.546 \\
\hline Chiguayante & Chiguayante & 3 & 3 & 72.454 \\
\hline Conchalí & Conchalí & 4 & 4 & 126.007 \\
\hline Concón & Concón & 1 & 1 & 38.419 \\
\hline Copiapó & Copiapó & 8 & 8 & 135.953 \\
\hline El Monte & El Monte & 1 & 1 & 34.101 \\
\hline La Cisterna & La Cisterna & 2 & 2 & 77.560 \\
\hline La Florida & La Florida & 9 & 9 & 331.976 \\
\hline La Reina & La Reina & 2 & 2 & 39.657 \\
\hline Lo Prado & Lo Prado & 4 & 4 & 140.990 \\
\hline Lota & Lota & 2 & 2 & 38.250 \\
\hline Padre Hurtado & Padre Hurtado & 1 & 1 & 50.460 \\
\hline Panquehue & Panquehue & 1 & 1 & 7.316 \\
\hline Peñaflor & Peñaflor & 2 & 2 & 76.787 \\
\hline Quilpué & Quilpué & 5 & 5 & 149.859 \\
\hline Rinconada & Rinconada & 1 & 1 & 9.541 \\
\hline San Joaquín & San Joaquín & 3 & 3 & 94.682 \\
\hline San Miguel & San Miguel & 2 & 2 & 87.879 \\
\hline San Pedro de la Paz & San Pedro de la Paz & 4 & 4 & 87.334 \\
\hline Villa Alemana & Villa Alemana & 3 & 3 & 91.131 \\
\hline
\end{tabular}


mente, se agrupan los pesos relativos generales por patología, conformando bandas de consumo similares, para lo que se utilizan los RUB. En la Tabla 3 aparecen los resultados para el agregado de patologías en todas las comunas consideradas en la muestra.

En términos generales, se observa que es mayor la prevalencia de pacientes con HTA $(57,051)$, seguida de diabetes $(26,868)$ e insuficiencia cardiaca $(5,211)$. Este patrón se invierte al analizar la morbilidad relativa, donde los pacientes con insuficiencia cardiaca son los que presentan el peso relativo mayor $(3,688)$, seguido de los pacientes con diabetes $(3,261)$ e HTA $(3,206)$. El comportamiento interno de los pesos relativos diferenciados por RUB muestra comportamientos similares entre las tres patologías con los mínimos oscilando en 1,9 y los máximos entre 5,2 y 5,4.

El comportamiento de las patologías para cada comuna, se presenta en las Tablas 4, 5 y 6.

Tabla 3. Comparación de prevalencia, morbilidad relativa agregada y distribución de pesos en RUB para el agregado de la muestra con las patologías crónicas seleccionadas

\begin{tabular}{|lccccc|}
\hline Patologías & $\begin{array}{c}\text { Prevalencia total } \\
(\mathbf{x ~ 1 . 0 0 0 )}\end{array}$ & $\begin{array}{c}\text { Morbilidad } \\
\text { relativa }\end{array}$ & $\mathbf{1}$ & $\mathbf{2}$ & $\mathbf{2}$ \\
\hline Diabetes & 26,868 & 3,261 & 1,917 & 3,304 & 5,326 \\
\hline HTA & 57,051 & 3,206 & 1,924 & 3,287 & 5,290 \\
\hline Ins. cardíaca & 5,211 & 3,688 & 1,820 & 3,444 & 5,443 \\
\hline
\end{tabular}

Tabla 4. Comparación de prevalencia, morbilidad relativa agregada y distribución de pesos en RUB por comuna, para el subgrupo de pacientes con diabetes mellitus

\begin{tabular}{|c|c|c|c|c|c|}
\hline \multirow[t]{2}{*}{ Comuna } & \multirow{2}{*}{$\begin{array}{l}\text { Prevalencia total } \\
(\text { (x 1.000) }\end{array}$} & \multirow{2}{*}{$\begin{array}{c}\text { Morbilidad } \\
\text { relativa }\end{array}$} & \multicolumn{3}{|c|}{ RUB } \\
\hline & & & 1 & 2 & 3 \\
\hline Caldera & 39,165 & 3,128 & 1,879 & 3,224 & 5,137 \\
\hline Catemu & 12,305 & 2,090 & 1,988 & 3,000 & 4,716 \\
\hline Cerro Navia & 32,858 & 3,489 & 1,862 & 3,275 & 5,373 \\
\hline Chiguayante & 3,202 & 1,639 & 2,105 & 2,989 & 5,504 \\
\hline Conchalí & 36,855 & 3,380 & 2,052 & 3,297 & 5,312 \\
\hline Concón & 29,517 & 3,819 & 1,952 & 3,323 & 5,422 \\
\hline Copiapó & 23,302 & 2,783 & 1,976 & 3,227 & 5,052 \\
\hline El Monte & 23,079 & 2,752 & 2,111 & 3,171 & 5,493 \\
\hline La Cisterna & 0,361 & 2,989 & 1,185 & 3,317 & 5,727 \\
\hline La Florida & 37,946 & 3,624 & 1,933 & 3,402 & 5,362 \\
\hline La Reina & 35,126 & 2,077 & 1,566 & 3,237 & 4,894 \\
\hline Lo Prado & 36,031 & 3,344 & 2,074 & 3,310 & 5,327 \\
\hline Lota & 8,026 & 2,287 & 2,141 & 3,138 & 3,569 \\
\hline Padre Hurtado & 29,945 & 2,527 & 2,205 & 3,153 & 4,843 \\
\hline Panquehue & 16,676 & 2,353 & 2,157 & 3,084 & 3,944 \\
\hline Peñaflor & 33,365 & 3,255 & 2,107 & 3,242 & 5,309 \\
\hline Quilpué & 16,516 & 3,172 & 2,069 & 3,248 & 5,156 \\
\hline Rinconada & 13,940 & 2,377 & 2,158 & 3,237 & 0,000 \\
\hline San Joaquín & 38,202 & 3,509 & 1,949 & 3,334 & 5,382 \\
\hline San Miguel & 27,515 & 1,053 & 0,764 & 3,132 & 4,936 \\
\hline San Pedro de la Paz & 11,828 & 2,505 & 1,939 & 3,208 & 4,856 \\
\hline Villa Alemana & 31,076 & 2,785 & 2,198 & 3,269 & 5,214 \\
\hline
\end{tabular}


Tabla 5. Comparación de prevalencia, morbilidad relativa agregada y distribución de pesos en RUB por comuna para el subgrupo de pacientes identificados con hipertensión arterial

\begin{tabular}{|lccccc|}
\hline Comuna & $\begin{array}{c}\text { Prevalencia total } \\
(\mathbf{x} 1.000)\end{array}$ & $\begin{array}{c}\text { Morbilidad } \\
\text { relativa }\end{array}$ & $\mathbf{1}$ & $\mathbf{2}$ ( & $\mathbf{3}$ \\
\hline Caldera & 60,473 & 3,133 & 1,917 & 3,260 & 5,155 \\
\hline Catemu & 28,309 & 2,395 & 1,884 & 3,107 & 5,105 \\
\hline Cerro Navia & 61,789 & 3,431 & 1,977 & 3,316 & 5,374 \\
\hline Chiguayante & 3,823 & 2,584 & 2,098 & 3,130 & 5,631 \\
\hline Conchalí & 74,234 & 3,373 & 2,062 & 3,308 & 5,286 \\
\hline Concón & 80,221 & 3,590 & 1,892 & 3,255 & 5,312 \\
\hline Copiapó & 37,307 & 2,893 & 2,030 & 3,257 & 5,149 \\
\hline El Monte & 61,376 & 2,836 & 2,165 & 3,189 & 5,255 \\
\hline La Cisterna & 0,670 & 3,024 & 1,395 & 3,075 & 5,580 \\
\hline La Florida & 86,606 & 3,472 & 1,925 & 3,312 & 5,295 \\
\hline La Reina & 55,072 & 2,536 & 1,650 & 3,162 & 4,932 \\
\hline Lo Prado & 69,409 & 3,376 & 2,093 & 3,328 & 5,313 \\
\hline Lota & 11,922 & 2,484 & 2,160 & 3,106 & 4,417 \\
\hline Padre Hurtado & 67,717 & 2,565 & 2,221 & 3,140 & 4,957 \\
\hline Panquehue & 36,495 & 2,465 & 2,141 & 3,104 & 3,686 \\
\hline Peñaflor & 72,603 & 3,296 & 2,103 & 3,272 & 5,337 \\
\hline Quilpué & 43,714 & 3,208 & 2,036 & 3,256 & 5,159 \\
\hline Rinconada & 22,220 & 2,575 & 2,210 & 3,034 & $N A$ \\
\hline San Joaquín & 83,163 & 3,443 & 1,878 & 3,314 & 5,321 \\
\hline San Miguel & 39,259 & 1,382 & 0,723 & 3,108 & 5,080 \\
\hline San Pedro de la Paz & 18,229 & 2,639 & 1,856 & 3,150 & 4,945 \\
\hline Villa Alemana & 65,302 & 2,850 & 2,210 & 3,305 & 5,191 \\
\hline
\end{tabular}

Es importante destacar la gran variabilidad de prevalencias observadas entre las comunas para las tres enfermedades. En el caso de diabetes mellitus, ésta va desde 0,361 por $1.000(0,036 \%)$ en La Cisterna a 39,165 (3,91\%) en Caldera (Tabla 4). En materia de HTA, la variación va de 3,823 $(0,38 \%)$ en Chiguayante a $83,16(8,31 \%)$ en San Joaquín (Tabla 5) y para insuficiencia cardíaca la variación va de 0,340 en Lota a 8,190 en Conchalí.

Si se considera que las prevalencias de diabetes y de HTA en la población general adulta son de 9,4\% y $26,9 \%$, de acuerdo a la Encuesta Nacional de Salud de 2009-2010 , llama la atención la gran distancia entre las prevalencias obtenidas a partir de los registros de población atendida y las que se esperarían si la totalidad de los pacientes existentes fueran detectados y tratados. Si bien es posible que las cifras obtenidas desde la población atendida se reduzcan por tratarse de una población general (con fuerte presencia infantil), la integridad del control de enfermedades crónicas se demuestra preocupante.

El análisis de las RUB, muestra que, con independencia de la prevalencia, las variaciones intercomunales son de menor cuantía que las que se aprecian en la prevalencia y morbilidad relativa.

\section{Discusión}

Los resultados muestran que tanto las prevalencias como la carga de morbilidad e intensidad de uso de recursos varían entre las distintas comunas estudiadas. De esta observación se desprenden 
Tabla 6. Comparación de prevalencia, morbilidad relativa agregada y distribución de pesos en RUB para el subgrupo de pacientes identificados con insuficiencia cardíaca

\begin{tabular}{|lccccc|}
\hline Comuna & $\begin{array}{c}\text { Prevalencia total } \\
(\mathbf{x ~ 1 . 0 0 0 )}\end{array}$ & $\begin{array}{c}\text { Morbilidad } \\
\text { relativa }\end{array}$ & $\mathbf{1}$ & $\mathbf{2}$ & $\mathbf{2}$ \\
\hline Caldera & 7,395 & 3,490 & 1,844 & 3,510 & 5,203 \\
\hline Catemu & 1,448 & 2,056 & 1,381 & 2,639 & 4,716 \\
\hline Cerro Navia & 10,299 & 3,892 & 1,905 & 3,483 & 5,450 \\
\hline Chiguayante & 0,262 & 2,762 & 2,046 & 2,925 & 5,336 \\
\hline Conchalí & 8,190 & 3,763 & 2,108 & 3,406 & 5,344 \\
\hline Concón & 4,399 & 4,359 & 1,759 & 3,465 & 5,752 \\
\hline Copiapó & 3,472 & 3,118 & 1,954 & 3,405 & 5,270 \\
\hline El Monte & 10,469 & 2,923 & 2,121 & 3,175 & 5,232 \\
\hline La Cisterna & 0,039 & 3,848 & $N A$ & 0,556 & 5,493 \\
\hline La Florida & 7,062 & 3,972 & 1,817 & 3,519 & 5,490 \\
\hline La Reina & 6,052 & 2,866 & 1,713 & 3,221 & 4,578 \\
\hline Lo Prado & 8,689 & 3,920 & 2,035 & 3,475 & 5,589 \\
\hline Lota & 0,340 & 2,783 & 1,386 & 3,304 & 4,716 \\
\hline Padre Hurtado & 1,130 & 2,682 & 2,181 & 3,135 & 5,957 \\
\hline Panquehue & 1,093 & 2,550 & 2,268 & 3,020 & $N A$ \\
\hline Peñaflor & 4,558 & 3,696 & 1,977 & 3,380 & 5,490 \\
\hline Quilpué & 2,189 & 3,715 & 1,904 & 3,436 & 5,216 \\
\hline Rinconada & 2,734 & 3,668 & $N A$ & 2,668 & $N A$ \\
\hline San Joaquín & 8,185 & 1,810 & 0,797 & 3,317 & 5,020 \\
\hline San Miguel & 3,676 & 2,932 & 1,872 & 3,298 & 4,860 \\
\hline San Pedro de la Paz & 1,969 & 3,906 & 2,104 & 3,665 & 5,386 \\
\hline Villa Alemana & 1,427 & & & \\
\hline
\end{tabular}

dos lecturas. La primera justifica estudios dirigidos a identificar la existencia de variables no médicas que pudieran estar determinando una mayor prevalencia de determinadas condiciones para actuar sobre estas con mayor efectividad. La segunda obliga a evaluar la efectividad de las estrategias de prevención secundaria y de cuidado por parte de los centros de salud, para implementar los cambios necesarios.

Un sistema de pago capitado, donde no se vincula el pago a un sistema de ajuste de según carga de morbilidad, como el vigente en Chile, centra la mayor parte del riesgo de financiamiento en el prestador, pues entrega la misma cantidad de recursos a un grupo de individuos sin considerar las necesidades intrínsecas de su condición de morbilidad ${ }^{10}$. Una mejora crítica es implementar un sistema de ajuste de riesgo basado en carga de morbilidad con el que sea posible conocer los gastos en los que se va a incurrir en atender a pacientes con un determinado perfil de morbilidad y distribuir los recursos de manera más equitativa. El ajuste también permite evaluar de mejor forma los resultados asociados a las intervenciones en salud para programas específicos, lo que facilita la rendición de cuentas, la evaluación de impacto y la transparencia a nivel de los proveedores, estimulando la calidad de la atención. La aplicación de ajustes de riesgo requiere conocer el perfil de morbilidad de la población, especialmente en lo que respecta a la prevalencia de enfermedades crónicas.

Herramientas como ACG, que permitan clasificar por carga de morbilidad a la población, tienen 
el potencial de mejorar la asignación de recursos concentrándose en la atención de aquellos cuya condición así lo requiera. Mejores sistemas de distribución de recursos son claves en el avance hacia un sistema de provisión de salud más eficiente, en especial en un sector tan importante como el sistema de Atención Primaria de Salud.

\section{Referencias}

1. Gottret P, Schieber G. Estudio del financiamiento de la salud: una guía para especialistas. Serie de estudios del Banco Mundial. 2007.

2. Roberts M, Hsiao W, Berman P, Reich M. Chapter 9; Payment Systems and Their Incentives. En: Getting Health Reform Right. Oxford University Press. 2008.

3. Duarte D. Asignación de Recursos per cápita en la Atención Primaria. La experiencia chilena. Serie Documentos de Estudio N 8. CPU, Santiago. 1995.

4. Montero J, Poblete F, Torche A, Vargas V. Análisis del modelo de asignación financiera en la atención primaria chilena: pertinencia del per-cápita actual y uso de variables en su cálculo para asegurar concordancia entre la situación epidemiológica actual y el modelo de atención. Centro de Políticas Públicas Universidad Católica. Propuestas para Chile 2008; 73-94.

5. Encuesta Nacional de Salud. Pontificia Universidad Católica de Chile. Ministerio de Salud. 2010. Disponible en: http://medicina.uc.cl/docman/1244/20/cat-view/ página-5.

6. Santelices E, Muñiz P, Arriagada I, Delgado M, Rojas J. Primera experiencia chilena con la aplicación de grupos clínicos ajustados (ACG) como herramienta de ajuste de riesgo: evaluación de la distribución de recursos en programa de enfermedades crónicas. Rev Med Chile 2014; 142 (1): 153-60.

7. The Johns Hopkins ACG $^{\circledR}$ System. Technical Reference Guide. Version 9.0. Johns Hopkins University, Bloomberg School of Public Health. 2009. Disponible en: http://acg.jhsph.org.

8. Santelices E, Rojas J, Delgado M, Arriagada L, Muñiz P. Distribución de la carga de morbilidad en Chile determinada por el Sistema ACG. En: XXXI Jornadas chilenas de salud pública. 2013. Disponible en: http:// www.saludpublica.uchile.cl.

9. Encuesta Nacional de Salud ENS Chile 2009-2010. Ministerio de Salud, Pontificia Universidad Católica de Chile, Universidad Alberto Hurtado. 2010. Disponible en: http://www.dinta.cl/wp-dintacl/wp-content/uploads/ Encuesta-Nacional-de-Salud-2010.pdf.

10. Cid C, Castillo C, Poblete S. Revisión de la literatura y sistematización de la información disponible sobre la asignación de recursos a nivel comunal. Estudios. Ministerio de Salud. 2012. Disponible en: http://www. medwave.cl/medios/medwave/InicTran/PDF/Recursos. comunal.pdf. 